\title{
Alkali-Induced Enhancement of Surface Electronic Polarizibility
}

\author{
Sergey Stolbov \\ Department of Physics, Cardwell Hall, Kansas State University, Manhattan, Kansas 66506, USA \\ Talat S. Rahman \\ Department of Physics, Cardwell Hall, Kansas State University, Manhattan, Kansas 66506, USA \\ and Fritz-Haber-Institut der Max-Planck-Gesellschaft, Faradayweg 4-6, D-14295 Berlin, Germany
}

(Received 7 June 2005; published 8 May 2006)

\begin{abstract}
From results of $a b$ initio electronic structure calculations based on density functional theory for a set of prototype systems, we find alkali adsorbates to cause a dramatic enhancement of the electronic polarizability of the metal surface extending it several angstroms into the vacuum. This phenomenon is traceable to an unusual feature induced in the surface potential on alkali adsorption. The effect appears to be general, as we find it to be present on metals as varied as $\mathrm{Pd}$ and $\mathrm{Cu}$, and helps explain the observed substantial decrease in the vibrational frequency of molecules when coadsorbed with alkalis on metal surfaces. Specifically, for two dissimilar molecules $\mathrm{CO}$ and $\mathrm{O}_{2}$, we trace the softening of the frequencies of their stretching mode when coadsorbed with $\mathrm{K}$ on $\mathrm{Pd}(111)$ to the enhanced electronic polarizability.
\end{abstract}

DOI: 10.1103/PhysRevLett.96.186801

PACS numbers: 73.20. $-\mathrm{r}, 71.15 .-\mathrm{m}$

Adsorption of alkalis on transition or noble metal surfaces dramatically changes the properties of the latter, making these systems the subject of extensive studies in areas extending from fundamental physics to chemical engineering. First, alkalis are known as efficient promoters in heterogeneous catalysis. The enhancement of reactivity under alkali adsorption has been reported for catalysts ranging from noble and transition metals with varying number of valence $d$ electrons, and oxides [1-4]. The effect extends to various types of reactions, such as dissociation of $\mathrm{O}_{2}, \mathrm{NO}, \mathrm{N}_{2}$, and $\mathrm{CH}_{4}$, oxidation of $\mathrm{CO}$, and methanol synthesis [1-3]. A related observation is a softening of the internal vibrational modes of molecules adsorbed on metal surface upon alkali coadsorption. As reported, the stretching mode of $\mathrm{CO}$ adsorbed on $\mathrm{Ru}(0001)$ was lowered from $2033 \mathrm{~cm}^{-1}$ to $1637 \mathrm{~cm}^{-1}$ upon Cs coadsorption [5], and to $1465 \mathrm{~cm}^{-1}$ upon $\mathrm{K}$ coadsorption [6]. Similar effect has been observed for $\mathrm{CO} / \mathrm{Ni}(111)$ [3], as well as for the stretching mode of NO adsorbed on $\mathrm{Pd}(111)$, upon K coadsorption [7]. This softening is thus found for metals with differing numbers of valence electrons, crystal structures, and surface geometries, indicating a generality of the effect. Since phonon softening usually originates from the weakening of intramolecular bonds, which make conditions favorable for further reactions, it is conceivable that both the promotion and the softening have the same driving force. Existing theoretical models have focused on relating the alkali promotion effect to the reduction of the work function [1], to the presence of the electric field associated with the surface barrier [8,9], and/or to the depopulation of surface states [10]. Interesting as these model calculations are, they do not provide a consistent picture of how changes in the surface electronic structure on alkali adsorption affect the frequencies of the internal modes of coadsorbed molecules. On the other hand, an understanding of the mechanism of the vibrational softening may provide insight also into the factors controlling surface reactivity, since intramolecular vibrations reflect the nature of interatomic bonds. In the literature, the vibrational softening of coadsorbed CO is mostly attributed to the enhanced back donation of the valence charge density from the metal to the molecule [6,11]. However, this model based on essentially a local concept of hybridization, cannot explain the long-range character of effects revealed in experimental observations: He and Jacobi [12] find that even if $\mathrm{CO}$ is adsorbed on the Ru surface far from the alkali atoms, the $\mathrm{C}-\mathrm{O}$ stretching mode is substantially softened. In their examination of the effect of alkali adsorption on hydrogen dissociation, Brown, et al. [13] also had to introduce the notion of long-range electronic perturbation in the vicinity of alkali-adsorbed metal surface to explain their observations.

It is clear from the above that mechanisms responsible for vibrational mode softening are nonlocal. The question is: what can give rise to this nonlocality? One possible answer is the electronic response of the metal [14]. Not only is the electronic response (polarizability) nonlocal, its enhancement is known to lead to phonon softening. For an isotropic system we can formally write

$$
\omega_{q}^{2}=\Omega_{q}^{2}[1-v(q) \chi(q)]
$$

where $\omega_{q}$ is the frequency of a phonon with wave vector $q$, $\Omega$ is its "bare" frequency obtained by neglecting the electronic response, $v(q)$ represents electron-electron interaction, and $\chi(q)$ is the electronic polarizability. More simply, Eq. (1) states that the perturbation induced by vibrations is screened by the electronic response. The larger the response is, the stronger is the screening, resulting in reduced force constants and, hence, lowered vibra- 
tional frequencies. This effect is well described in textbooks [15] and a nice illustration can be found in Ref. [16].

In this Letter, we show that, indeed, the effect of alkali adsorption is to produce a pronounced increase in the surface electronic polarizability whose origin we trace to a characteristic feature induced in the surface potential barrier. To establish that this phenomenon is general we have carried out calculations for both $\mathrm{K}$ and $\mathrm{Na}$ adsorbed on the surfaces of two metals, $\mathrm{Pd}$ and $\mathrm{Cu}$, whose properties differ in several ways, not the least of which is the difference in the density of states at the Fermi level. Furthermore, the vibrational dynamics of two chemically distinct molecules $\mathrm{CO}$ and $\mathrm{O}_{2}$ are shown to display a substantial lowering of their frequencies on coadsorption with $\mathrm{K}$ on $\mathrm{Pd}(111)$. To our knowledge such a microscopic theoretical investigation has not yet been carried out.

Our calculations are based on self-consistent electronic structure calculations carried out using the full-potential linearized augmented plane wave (FLAPW) method [17] as embodied in the WIEN2K code [18]. We mimic the surface system with a supercell consisting of 8 atomic layers of $\mathrm{Cu}$ or Pd and $11 \AA$ thick vacuum. Calculations have been performed for two-dimensional $(2 \times 2)$ unit cells with 4 metal atoms per layer, plus one alkali atom on each side of the slab, corresponding to 1/4 ML coverage of the alkali. A fragment of the surface with the adsorbate is shown in Fig. 1. To check the effect of alkali coverage, calculations have been repeated with a $(3 \times 2)$ unit cell corresponding to $1 / 6 \mathrm{ML}$ alkali coverage. The two-dimensional surface Brillouin zone is sampled by 25 and $16 k$ points for the $(2 \times 2)$ and $(3 \times 2)$ superstructures, respectively. The exchange-correlation part of the potential is calculated in the local density approximation within the

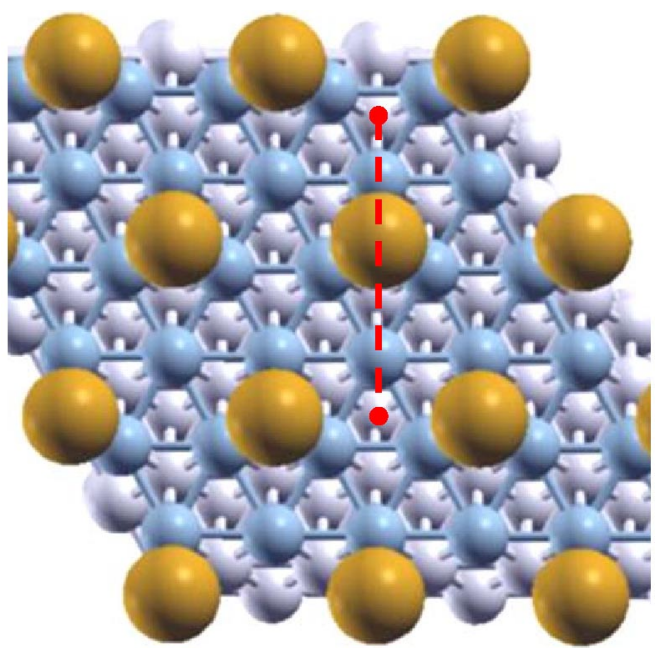

FIG. 1 (color online). Top view of the $A_{0.25} / \mathrm{M}(111)$ surface $(A=\mathrm{Na}, \mathrm{K} ; \mathrm{M}=\mathrm{Cu}, \mathrm{Pd})$. The large balls represent alkali atoms, small dark balls are for the first layer $\mathrm{M}$ atoms and small gray balls are for the rest of the $\mathrm{M}$ atoms. The dashed line represents the projection of the plane along which $w^{N}(r)$ is plotted in Fig. 2. density functional theory [19]. Frequencies of C-O and O$\mathrm{O}$ stretching mode are calculated within the frozen-phonon approximation [20].

Since direct calculation of the electronic polarizability in the vicinity of surfaces is not feasible, we turn to the calculation of a related quantity, the isoelectronic reactivity index $w^{N}(r)$ introduced by Wilke et al. [21]:

$$
w^{N}(r)=\frac{1}{k^{2} T_{\mathrm{el}}}\left[\frac{\partial \rho\left(r, T_{\mathrm{el}}\right)}{\partial T_{\mathrm{el}}}\right]_{V(r), N} \approx \frac{\rho\left(r, T_{\mathrm{el}}\right)-\rho(r, 0)}{\left(k T_{\mathrm{el}}\right)^{2}} .
$$

Here $\rho(r)$ is the electronic density, $V(r)$ denotes an external potential, $N$ is the number of electrons in the system, $k$ is Boltzmann's constant, and $T_{\mathrm{el}}$ is the electronic temperature allowing a convolution of the electronic states with the Fermi-Dirac function for a given temperature. In this study we set $k T_{\text {el }}=0.01 \mathrm{Ry}$. The physical interpretation of $w^{N}(r)$ is as follows. An increase in $T_{\mathrm{el}}$ from " 0 " to a finite value introduces electronic excitations (electron-hole pairs) obeying the Fermi-Dirac distribution. These excitations cause an electronic density variation which is reflected in the last term of Eq. (2). Since creation of the electron-hole pairs is a natural response to an external perturbation (variation of potential), $w^{N}(r)$ characterizes the electronic polarizability. Note that the isoelectronic reactivity index is also closely related to the local softness function $s(r)$ [22] which is widely used in quantum chemistry as a measure of the electronic response of the system [23].

In Fig. 2, two-dimensional plots of $w^{N}(r)$ calculated for clean $\operatorname{Pd}(111)$ and $\mathrm{K}_{0.25} / \mathrm{Pd}(111)$ show that adsorption of potassium induces a huge increase in $w^{N}(r)$ in the vacuum region. And more to the point here, the effect is nonlocal, since $w^{N}(r)$ is substantially enhanced not only around the adsorbate atom, but also for the entire surface area. A similar and even larger effect is found for a 1/4 ML of $\mathrm{Na}$ on $\mathrm{Cu}(111)$. More quantitatively, the effect can be illustrated by one-dimensional plots of $w^{N}(r)$ in Fig. 3, along the surface normal passing through the fcc hollow
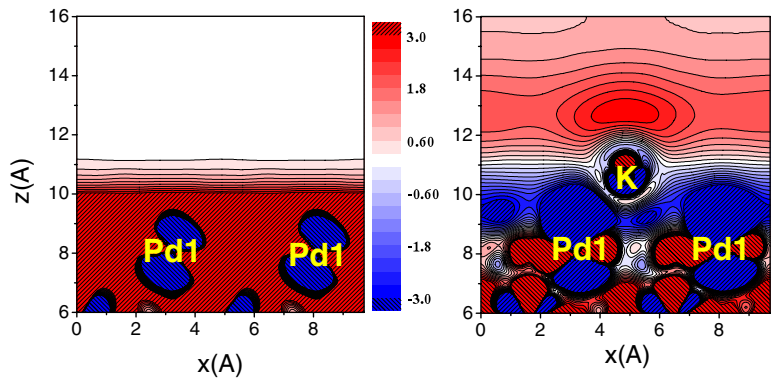

FIG. 2 (color online). Plot of $w^{N}(r)$ for clean $\mathrm{Pd}(111)$ (left panel) and for $\mathrm{K}_{0.25} / \mathrm{Pd}(111)$ (right panel) along the plane perpendicular to the surface. The Pd1 labels mark the positions of the topmost $\mathrm{Pd}$ atoms and $\mathrm{K}$ that of potassium atoms. A drastic decrease in $w^{N}(r)$ is seen for the clean surface [the white area corresponds to $w^{N}(r)=0$, while adsorption of K induces a huge increase and delocalization of $w^{N}(r)$ even far from the adsorbate]. 

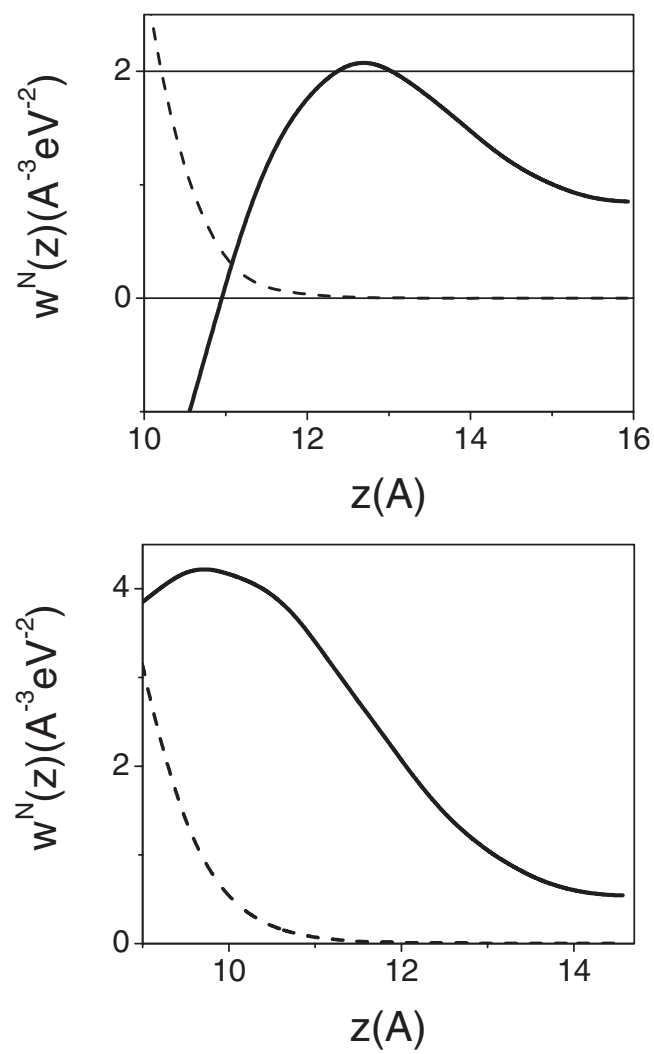

FIG. 3. Plot of $w^{N}(r)$ along the surface normal. The upper panel shows $w^{N}(r)$ for $\operatorname{Pd}(111)$ (dashed line) and $\mathrm{K}_{0.25} / \mathrm{Pd}(111)$ (solid line). The lower panel shows $w^{N}(r)$ for $\mathrm{Cu}(111)$ (dashed line) and $\mathrm{Na}_{0.25} / \mathrm{Cu}(111)$ (solid line).

site, which is maximally distant from the adsorbate atoms. Clearly, $w^{N}(r)$ decays rapidly at the clean surfaces, whereas, upon alkali adsorption, it is increased and delocalized over 2-4 $\AA$ away from the surface. Such behavior of $w^{N}(r)$ implies that alkali adsorption dramatically enhances the electronic polarizability in the vacuum region in the vicinity of the surface.

For insights into the microscopic basis for these dramatic effects we have calculated the total self-consistent potential for the surfaces under consideration. Onedimensional plots of the potentials in Fig. 4 testify to the well-known reduction of the potential barrier at the surface which further manifests itself in a decrease in the work functions for $\operatorname{Pd}(111)$ and $\mathrm{Cu}(111)$. What is indeed very intriguing and novel is that instead of an expected simple reduction of the barrier, alkali adsorbates form a groovelike or plateaulike region of further reduced potential in the vicinity of the surface. Since this feature is energetically located around $E_{F}$, it is easy to see that it is responsible for the low-energy delocalized excited electronic states which, in turn, enhance the surface electronic polarizability.

We now turn to the role of the electronic polarizability in the softening of internal vibration modes of coadsorbed molecules. We consider two distinct cases- $\mathrm{CO}$ which prefers to bind vertically and $\mathrm{O}_{2}$ which orients its axis parallel to the surface. For $\mathrm{CO}$ adsorbed on top of a $\mathrm{Pd}$
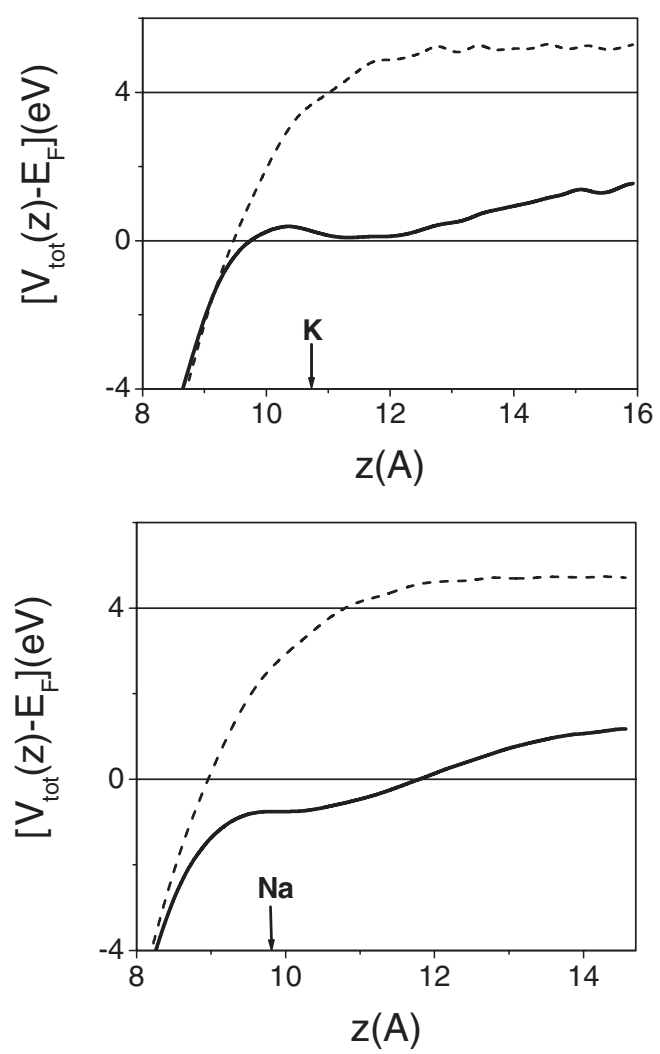

FIG. 4. The self-consistent total potential plotted along the surface normal. The upper panel displays the potential for $\operatorname{Pd}(111)$ (dashed line) and $\mathrm{K}_{0.25} / \mathrm{Pd}(111)$ (solid line). The lower panel provides the same for $\mathrm{Cu}(111)$ (dashed line) and $\mathrm{Na}_{0.25} / \mathrm{Cu}(111)$ (solid line). Arrows indicate the heights of the alkali atoms.

atom [1/4 ML coverage on $\operatorname{Pd}(111)]$, we find the frequency of the $\mathrm{C}-\mathrm{O}$ stretching mode $\left(f_{\mathrm{CO}}\right)$ to soften from 1990 to $1800 \mathrm{~cm}^{-1}$ on the presence of $\mathrm{K}$ on the surface. To separate the effect of the enhanced electronic polarizability on the softening, we move the molecule away from the surface increasing C-Pd distance from 1.84 to $3.3 \AA$. At such a location, C-Pd or C-K bonding is negligible. At the same time, $\mathrm{CO}$ is located in the region where the electronic polarizability is very low for clean $\mathrm{Pd}(111)$ and high for $\mathrm{K}_{0.25} / \mathrm{Pd}(111)$ (see Fig. 2). The calculations result in $f_{\mathrm{CO}}=2100 \mathrm{~cm}^{-1}$ for clean $\operatorname{Pd}(111)$ and $f_{\mathrm{CO}}=$ $1860 \mathrm{~cm}^{-1}$ for the surface with coadsorbed K. Thus breaking $\mathrm{Pd}-\mathrm{C}$ bond on clean $\mathrm{Pd}(111)$ reverts $\mathrm{C}-\mathrm{O}$ stretch frequency to almost the free molecule value $\left(2130 \mathrm{~cm}^{-1}\right)$, while coadsorbed $\mathrm{K}$ retains the softened frequency despite the lack of direct C-Pd bond. This result shows the direct link between vibrational mode softening and enhanced electronic polarizability.

Next, we calculate the frequency $\left(f_{\mathrm{OO}}\right)$ of the O-O stretching mode for $\mathrm{O}_{2}$ placed above both clean $\mathrm{Pd}(111)$ and $\mathrm{K}_{0.25} \mathrm{Pd}(111)$. Interestingly, $\mathrm{O}_{2}$ can be a perfect probe for the effect of electronic polarizability on vibrational frequencies since we can eliminate not only chemical bonding effects (as we did above for $\mathrm{CO}$ ), but also possible 
electric field effects. We place $\mathrm{O}_{2}$ parallel to the $\mathrm{Pd}(111)$ plane $4.5 \AA$ above the surface such that for $\mathrm{K}_{0.25} / \mathrm{Pd}(111)$, the oxygen atoms are maximally distant from the adsorbed $\mathrm{K}$ (O-Pd and O-K distances are 4.7 and $3.3 \AA$, respectively) and there is no direct $\mathrm{K}-\mathrm{O}$ bond. Our calculations show that there is no force acting on $\mathrm{O}_{2}$ from $\mathrm{K}$ or Pd atoms and there is no noticeable difference in the potential on the two oxygen atoms, thus excluding the presence of an electric field. This is understandable since the oxygen molecule lies parallel to the surface. Besides, in contrast to $\mathrm{CO}, \mathrm{O}_{2}$ does not have any dipole moment and, hence, does not produce any long-range electrostatic interaction. Consequently, the surface can affect the properties of $\mathrm{O}_{2}$ only through the electronic response. We find that $f_{\mathrm{OO}}=1550 \mathrm{~cm}^{-1}$ for $\mathrm{O}_{2}$ placed above clean $\operatorname{Pd}(111)$, which is almost the vibrational frequency for a free molecule $\left(1560 \mathrm{~cm}^{-1}\right)$, while $f_{\mathrm{OO}}=1280 \mathrm{~cm}^{-1}$ for the surface with coadsorbed $\mathrm{K}$. Rotation of the molecule around a surface normal by $45^{\circ}$ hardly changes the frequency $\left(1250 \mathrm{~cm}^{-1}\right)$ suggesting isotropy of the $\mathrm{O}_{2}$ environment and hence confirming the absence of any chemical bonding to $\mathrm{Pd}$ or $\mathrm{K}$ atoms. Reduction of the $\mathrm{K}$ coverage from $1 / 4 \mathrm{ML}$ to $1 / 6 \mathrm{ML}$ only slightly reduces the polarizability in the vicinity of the surface related to a small $(0.2 \mathrm{eV})$ increase in the work function. For the lower coverage we find $f_{\mathrm{OO}}=1330 \mathrm{~cm}^{-1}$ when the $\mathrm{O}_{2}$ molecule is $4.5 \AA$ above the Pd surface. Although with the reduction of the $\mathrm{K}$ coverage the $\mathrm{K}-\mathrm{O}$ distance is substantially increased (from 3.3 to $3.9 \AA$ ), $f_{\mathrm{OO}}$ is still significantly lower than that for the clean Pd surface. These results clearly show that the strong enhancement of electronic polarizability of the metal surface upon alkali adsorption is the driving force for the softening of the internal vibrational modes observed for coadsorbed molecules.

We find that apart from the softening of vibrational frequencies of $\mathrm{O}_{2}$ placed above $\mathrm{Pd}(111), \mathrm{K}$ coadsorption causes an increase in the $\mathrm{O}-\mathrm{O}$ bond length from 1.23 to $1.30 \AA$ and a decrease in the O-O bonding energy from 7.9 to $5.2 \mathrm{eV}$, as obtained from calculations for the $1 / 4 \mathrm{ML}$ coverage of $\mathrm{K}$. These findings can also be traced to the enhancement of electronic polarizability upon alkali adsorption: enhanced electronic response screens the perturbation induced by the stretching of the molecule and helps weaken the $\mathrm{O}-\mathrm{O}$ bond, making conditions favorable for further reactions with other adsorbed molecules. In other words, the enhancement of the polarizability of the metal surfaces upon alkali adsorption is ultimately responsible for facilitating surface reactions. We are in the process of quantifying such effects for specific reactions. The general underlying mechanisms, however, have been captured in the examples here.

In summary, detailed first principles studies of the electronic structure of $\mathrm{Pd}(111)$ and $\mathrm{Cu}(111)$ reveal an unusual feature of the surface potential on alkali adsorption which causes a dramatic enhancement of the surface electronic polarizability extended toward the vacuum. We show that the frequencies of internal vibrational modes of molecules such as $\mathrm{CO}$ and $\mathrm{O}_{2}$ when coadsorbed with alkalis on these metal surfaces undergo remarkable softening, as a direct result of the enhanced surface electronic polarizablity. The effect is equally pronounced for metals as dissimilar as $\mathrm{Pd}$ and $\mathrm{Cu}$ and adsorbates as varied as $\mathrm{CO}$ and $\mathrm{O}_{2}$.

We thank G. Ertl, A. Luntz and K. Jacobi for helpful discussions, and acknowledge financial support from DOE under Grant No. DE-FGO3-03ER15464 and NSF under Grant No. CHE-0205064. T. S. R. would like to thank the Alexander von Humboldt Foundation for a "Forschungspreiz," and colleagues at the Fritz Haber Institute, Berlin, for their hospitality.

[1] G. Ertl, M. Weiss, and S. Lee, Chem. Phys. Lett. 60, 391 (1979).

[2] L. J. Whitman and W. Ho, J. Chem. Phys. 89, 7621 (1988).

[3] A. Cupolillo et al., Phys. Rev. B 66, 233407 (2002).

[4] M. Maack, H. Friis-Jensen, S. Scherl, J.H. Larsen, and I. Chorkendorff, Top. Catal. 22, 151 (2003).

[5] G. E. Thomas and W. H. Weinberg, J. Chem. Phys. 70, 954 (1979).

[6] F. M. Hoffmann and R. A. de Paola, Phys. Rev. Lett. 52, 1697 (1984).

[7] H. Orita, I. Nakamura, and T. Fujitani, Surf. Sci. 571, 102 (2004).

[8] N. D. Lang, S. Holloway, and J. K. Nørskov, Surf. Sci. 150, 24 (1985).

[9] J. J. Mortensen, B. Hammer, and J. K. Nørskov, Phys. Rev. Lett. 80, 4333 (1998).

[10] E. Bertel, P. Roos, and J. Lehmann, Phys. Rev. B 52, R14384 (1995).

[11] H. P. Bonzel, Surf. Sci. Rep. 8, 43 (1988).

[12] P. He and K. Jacobi, J. Chem. Phys. 106, 3417 (1997).

[13] J. K. Brown, A. C. Luntz, and P. A. Schultz, J. Chem. Phys. 95, 3767 (1991).

[14] L. J. Sham, Phys. Rev. 188, 1431 (1969).

[15] G. P. Srivastava The Physics of Phonons (Institute of Physics Publishing, Bristol, Philadelphia and New York, 1990).

[16] C. M. Varma and W. Weber, Phys. Rev. Lett. 39, 1094 (1977).

[17] D. Singh, Planewaves, Pseudopotentials and the LAPW Method (Kluwer Academic, Boston, 1994), ISBN No. 07923-9421-7.

[18] P. Blaha, K. Schwarz, G. K. H. Madsen, D. Kvasnicka, and J. Luitz, WIEN2K, An Augmented Plane Wave + Local Orbitals Program for Calculating Crystal Properties (Karlheinz Schwarz, Technische Universität Wien, Austria, 2001), ISBN No. 3-9501031-1-2.

[19] J. P. Perdew and Y. Wang, Phys. Rev. B 45, 13244 (1992).

[20] K. Kunc and R. M. Martin, Phys. Rev. Lett. 48, 406 (1982).

[21] S. Wilke, M. H. Cohen, and M. Scheffler, Phys. Rev. Lett. 77, 1560 (1996).

[22] R. G. Pearson, Science 151, 172 (1966).

[23] R. G. Parr and W. Yang, Density Functional Theory of Atoms and Molecules (Oxford University Press, New York, 1989). 